\title{
Das «Unfallähnliche Ereignis» und seine bedenklichen Folgen
}

\author{
Nach Einführung des «Unfallähnlichen Ereignisses» in der Unfallversicherung zeigt \\ sich in der Praxis die problematische Kehrseite einer Vereinfachung der Kausalitäts- \\ beurteilung. Es muss über Entscheidungsgrundlagen nachgedacht werden, die \\ wieder die pathomorphologischen und rudimentären traumabiomechanischen
} Aspekte miteinbeziehen.

Luzi Dubs ${ }^{a}$,

Marc-Daniel Zumstein ${ }^{b}$,

Bruno Soltermann',

Christoph Bosshard,

Josef E. Brandenberge

a Facharzt Orthopädische Chirurgie, Winterthur

b Facharzt Orthopädische Chirurgie, Aarau

c Facharzt Chirurgie, Chefarzt Schweizerischer Versicherungsverband SVV, Zürich

d Facharzt Orthopädische Chirurgie, Bern

e Facharzt Orthopädische Chirurgie, Ombudsmann fmCh, Luzern
Nach der Einführung des «Unfallähnlichen Ereignisses» in der Unfallversicherung (UVG) zeigt sich im Praxisalltag der Ärzteschaft immer deutlicher die Kehrseite einer aus juristischer Sicht verständlichen Vereinfachung der Kausalitätsbeurteilung. Die Bedeutungslosigkeit der Pathomorphologie hat zu einer bedenklichen Vernachlässigung der Dokumentation und die unterschiedlichen finanziellen Anreize haben zu einer Verzerrung bei der Beschreibung der medizinischen Realität geführt. Dass der Gesetzgeber die primäre Beurteilung der UnfallKausalität eines unfallähnlichen Ereignisses durch die Ärzteschaft nicht vorsieht, erschwert die Arbeit der später eingesetzten ärztlichen Gutachter, die sich im Nachhinein explizit zur Kausalität äussern müssen.

\section{Einleitung}

Seit 1984 existiert in der Schweiz ein Unfallversicherungsgesetz, das - einzigartig auf der Welt - den Begriff der «unfallähnlichen Körperschädigung UKS» verwendet. Der Bundesrat hat von seiner Kompetenz Gebrauch gemacht und auf Verordnungsebene eine abschliessende Liste solcher Diagnosen erstellt. Die Rechtsanwendung in ihrer heutigen Form wurde
Sofern sie nicht eindeutig auf eine Erkrankung oder eine Degeneration zurückzuführen sind, sind die unfallähnlichen Körperschädigungen den Unfällen gleichgestellt, auch wenn die ungewöhnliche äussere Einwirkung fehlt. Folgende Begriffsmerkmale prägen somit das unfallähnliche Ereignis: plötzliche, unbeabsichtigte/unfreiwillige und schädigende äussere Einwirkung auf den menschlichen Körper. Sie kann aber alltäglich und diskret sein, auch in einer körpereigenen Bewegung entstehen.

\section{Das sinnfällige Ereignis}

Die beim üblichen Unfallbegriff verlangte Ungewöhnlichkeit wird durch die Sinnfälligkeit ersetzt. In Folge des EVG-Urteils vom 5. Juni 2001 steht fest, dass bloss entscheidend ist, ob ein äusseres objektivierbares sinnfälliges Ereignis die Beschwerden verursacht, oder zumindest ausgelöst hat. Im konkreten Fall wurde die Unfallversicherung für einen eindeutig degenerativ veränderten Meniskusriss, der nach einem Sprung von einer Kiste schmerzhaft wurde, als zuständig betrachtet [2]. Die Entscheidung, ob ein sinnfälliges Ereignis vorliegt, ist eine Rechtsfrage.

\section{In der Praxis zirkuliert eine für Mediziner bei genauer Betrachtung äusserst widersprüchliche Liste mit Geschehnissen.}

Korrespondenz: Dr. med. Luzi Dubs Merkurstrasse 12 CH-8400 Winterthur Tel. 0522132223 Fax 0522125804 dubs.luzi[at]bluewin.ch durch das Urteil des Eidgenössischen Versicherungsgerichts (EVG) vom 5.6.2001 präzisiert. Der Unterschied zum eigentlichen Unfallbegriff wurde im gleichen Jahr in der Schweizerischen Ärztezeitung publiziert [1]. Verschiedene Beobachtungen geben Anlass zu fragen, ob sich diese Praxis aus medizinischer Sicht bewährt hat und Anreizverlagerungen entstanden sind. Die Rolle der Ärzteschaft, die sich im Rahmen der «Unfallähnlichen Körperschädigung» (UKS) nicht mehr zur Kausalität und zur Pathomorphologie zu äussern hat, ist zu hinterfragen.
In der Praxis zirkuliert eine für Mediziner bei genauer Betrachtung äusserst widersprüchliche Liste mit Geschehnissen, die entweder als Einschlussoder als Ausschlusskriterium gelten sollen.

\section{Die Listendiagnose}

Neben der Nennung eines sinnfälligen Ereignisses muss aufgrund der ärztlichen Abklärung eine Listendiagnose als Hauptdiagnose vorliegen. Gemäss Art. 9 Abs.2 UVV (gültig ab 1.1.1998) sind folgende, abschliessend aufgeführte Körperschädigungen, sofern 
sie nicht eindeutig auf eine vorbestehende Erkrankung oder eine Degeneration zurückzuführen sind, auch ohne ungewöhnliche äussere Einwirkung Unfällen gleichgestellt: Knochenbrüche, Verrenkungen von Gelenken, Meniskusrisse, Muskelrisse, Muskelzerrungen, Sehnenrisse, Bandläsionen, Trommelfellverletzungen [2].

Sind Listendiagnose und sinnfälliges Ereignis kombiniert anzutreffen, sind grundsätzlich die Voraussetzungen zur Übernahme durch die soziale Unfallversicherung erfüllt. Der zeitliche Zusammenhang zwischen dem sinnfälligen Ereignis und der Listendiagose sollte «innert vernünftiger Zeit» stehen, eine Kausalitätskette sollte erkennbar sein.

Die meisten Fälle eines unfallähnlichen Ereignisses betreffen Schäden des Meniskus. An diesem Beispiel lässt sich im Folgenden die entstandene Problematik aus medizinischer Perspektive gut diskutieren. lisierung unserer Aussagen eingeschränkt. Hiermit sind alle klinisch tätigen Berufsgruppen, speziell auch alle Radiologen, angesprochen.

Auch die Begriffe der vorliegenden Erkrankung und der Degeneration, die im Gesetzestext als Ausschlusskriterium für eine unfallähnliche Körperschädigung aufgeführt werden, jedoch durch das EVG-Urteil von 2001 relativiert wurden, müssten eigentlich in jeder Situation klarer definiert und diskutiert sein. Will man seitens der Medizin das Kriterium des Risses als erfüllt betrachten, müsste ein Riss im eigentlichen Sinne in gesundem Gewebe zustande gekommen sein, d.h., dieselbe akut gewebeschädigende Belastung müsste bei allen die gleiche Schädigung erzeugt haben bzw. die Rissform sollte zur Traumaintensität passen. Die Degeneration ist nicht allein eine Frage des Alters, die praktische Erfahrung zeigt nicht allzu selten bereits degenerative

\section{Der neutralere Begriff einer «Schädigung» wäre eher angebracht als der präjudizierende Begriff des «Risses».}

\section{Schädigung oder Riss - ein linguistischer Determinismus?}

Die Erfahrung zeigt immer wieder, dass in den weitaus häufigsten Fällen ein Untersucher weder im MRI noch anlässlich der Arthroskopie das morphologische Bild klar dem vermuteten Ereignis zuordnen kann. Daher wäre viel eher der neutralere Begriff einer «Schädigung» als der präjudizierende Begriff des «Risses» angebracht. Das Bild des Meniskusschadens ist meist komplex, und die Gewebsqualität mit den Erweichungen und den Gelbverfärbungen zeigt sehr häufig die Merkmale einer degenerativen Vorschädigung. Aus pathomorphologischer Sicht besteht oft der Eindruck, dass keine frische Schädigung, sondern eine erhöhte Anfälligkeit des Gewebes ausschlaggebend gewesen ist. Während ein gesundes Gewebe nur bei hoher Belastung reisst, führen bei degenerierten Strukturen schon übliche und geringe Alltagsbelastungen zu Schäden.

Solange der Begriff des «Risses» als Präjudiz weiterhin eingesetzt wird, bleibt von vorneweg der Eindruck der Traumarelevanz haften. Es besteht die Gefahr, einem linguistischen Determinismus zu erliegen. So wie man denkt, so wird gesprochen und demnach gehandelt.

Für Mediziner paradox anmutend spricht der Verordnungstext von Rissen am Meniskus, bei den Bändern hingegen, die viel eher reissen oder zerren und morphologisch klarer als Unfallfolgen in Erscheinung treten, spricht der Gesetzestext von Läsionen bzw. Schädigungen. Es ist somit von medizinischer Seite zu fordern, primär von einer Schädigung des Meniskus zu sprechen. Dadurch wird eine spätere Instrumenta-
Schädigungen bei Patienten vor dem Wachstumsabschluss, die z.B. durch gewisse Kampfsportarten oder intensive Belastungen vom Stop-and-go-Charakter exponiert sind.

\section{Kausalität in der Diagnostik}

Es gibt weltweit keine Studie, welche die Frage beantworten kann, ob der Rückenschmerz wegen eines Bandscheibenvorfalls oder der Bandscheibenvorfall wegen der Rückenschmerzen zustande gekommen ist (Post-hoc-ergo-propter-hoc-Bias). Es handelt sich jeweils um Querschnittsstudien mit Korrelationsaussagen, in denen prinzipiell keine Kausalität abgeleitet werden kann. Man kann lediglich von mehr oder weniger starken Zusammenhängen sprechen. Analog ist die Situation beim Kniegelenk. Wenn jemand plötzlich Knieschmerzen bekommt, sei es beim Aufstehen aus dem Kauern oder bei einer banalen Knieprellung, und die klinische sowie radiologische Abklärung eine Meniskusschädigung ergibt, heisst dies bei weitem nicht automatisch, dass Letztere wegen des Ersteren entstanden ist. Auch der Rückschluss, der bildgebend festgestellte Meniskusschaden sei Ursache für die Kniebeschwerden, ist nicht zwingend gegeben. Für die Anerkennung einer UKS sind aber lediglich der rechtliche Tatbestand eines sinnfälligen Ereignisses gemäss Kasuistikliste und der medizinische Nachweis eines Meniskusrisses ausschlaggebend. Die Forderung nach einer Kausalitätskette wird fragwürdig, wenn nicht klar ausgesagt werden kann, ob die Listendiagnose schon vor dem Ereignis hätte gestellt werden können. 


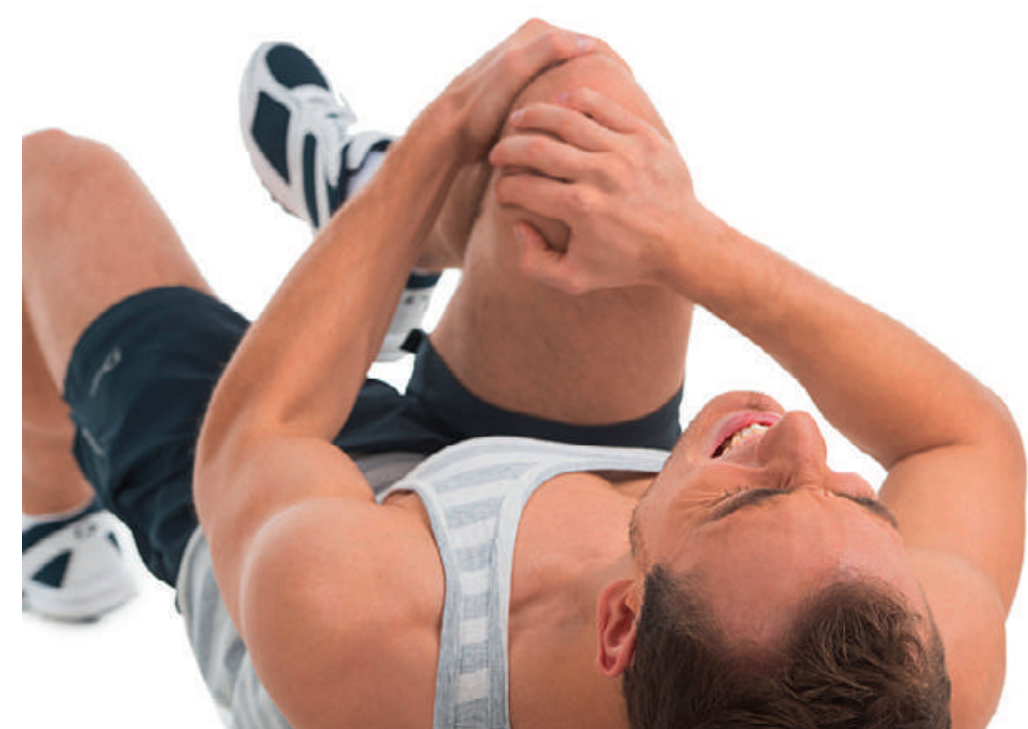

Das Ereignis und der Nachweis eines Meniskusrisses sind für die Anerkennung als «Unfallähnliche Körperschädigung» ausschlaggebend. Niemand fragt, ob das Knie schon vorgeschädigt war.
Krankheitsmanifestationen seien selbstredend Folgen einer UKS. Ist einmal die Zuständigkeit anerkannt, muss gemäss Gesetz der Unfallversicherer den Nachweis erbringen, dass es sich bei den weiteren Beschwerden nicht mehr um Unfallfolgen handelt. Ab diesem Moment sind wieder pathomorphologische Argumentationen gefragt. Die Ablehnung der weiteren Zuständigkeit ist weitaus schwieriger als die initiale Prüfung des Leistungsanspruchs zu einem Zeitpunkt, wo die Beweislast beim Versicherten liegt.

\section{Medizinische Verzerrungen durch Fehlanreize}

Es wird aus juristischer Warte eingeräumt, dass es sich beim unfallähnlichen Ereignis um ein «Zwischending» zwischen Unfall und Krankheit handelt, das bezweckt, die «bei bestimmten Verletzungen besonders schwer verständliche Grenzziehung zwischen Unfällen und Krankheiten und damit zwischen zwei sehr ungleich ausgestalteten Leistungssystemen ein wenig zugunsten der Versicherten zu verschieben»,... «da die Leistungen der sozialen Unfallversicherung viel besser sind als diejenigen der Krankenversicherung» [1]. Es ist der Wille des Gesetzgebers, im Rahmen der UKS-Diagnosen die Krankenversicherungen zu entlasten. Dieses Grundproblem der unterschiedlichen Systeme, mit den damit verbundenen Fehlanreizen in mehrere Richtungen, bleibt allerdings über die UKS-Frage hinaus bestehen. Das «Zwischending» UKS erleichtert aber die besagte Verschiebung ins UVG und die Entlastung der Krankenversicherungen erheblich.

Es wird wohl niemanden wundern, wenn dieses speziell in UKS-Fragen heikle Anreizsystem aus medizinethischen Überlegungen äusserst bedenkliche Folgen zeitigt. Es hat sich mittlerweile breitflächig bei Arbeitgebern und Patienten herumgesprochen, dass alles unternommen werden muss, den Graubereich der UKS auszunützen und - oftmals mit zeitlicher Verzögerung - ein kaum nachprüfbares unfallähnliches Ereignis zu formulieren, um von den Vorzügen des UVG zu profitieren. Die Kostenbeteiligung des KVG durch Franchise und Selbstbehalt fällt weg. Dem Arbeitgeber wird der Ausfall seines Arbeitnehmers durch die UVG-Versicherung in Form von Taggeldern schon nach kurzer Zeit finanziell abgegolten. Auch der behandelnde Arzt profitiert von meist höheren Taxpunktwerten im UVG. Und nicht selten hat der Arbeitgeber als Ergänzung zum UVG eine Zusatzversicherung für Leistungen bei Unfällen abgeschlossen.

Bezeichnenderweise können die Hausfrauen, die Kinder, die Studenten und die meisten Selbständigerwerbenden, insbesondere die Landwirte, die nicht UVG-versichert sind, diese Vorzüge nicht nutzen.

Schicksalsbedingter Unfallbegriff ist verwässert Der eigentliche Unfallbegriff mit dem ungewöhnlichen äusseren Faktor hat häufig mit dem Begriff des 
unberechenbaren Schicksals zu tun, speziell wenn ein Fremdverschulden vorliegt. Diese Besonderheiten werden im Vergütungssystem der Unfallversicherer berücksichtigt. Mit einer UKS hingegen begibt man sich in die Grauzone der plötzlich begonnenen Primärmanifestation einer Erkrankung. Aus juristischer Sicht mag die erhoffte Erleichterung in der Entscheidungsfindung durch Etablierung der UKS nachvollziehbar zu sein. Aus medizinischer, ökonomischer und ethischer Sicht sind aber neue Fehlanreize entstanden, welche die Qualität des Denkens und Handelns überstrapazieren, neue Interessenkonflikte erzeugen und die Tendenz begünstigen, im UKS-Fall gar keine Überprüfung der Kausali-

\section{Fazit}

Die Autoren gehen davon aus, dass die UKS in absehbarer Zeit nicht eliminiert werden kann, sondern als «Sonderfall Schweiz» weiterbestehen wird. Zwecks Verminderung der negativen Folgen seien Anregungen sowohl an die Ärzteschaft als auch an die Versicherer erlaubt:

- Die Sprachbegriffe sind neutral zu wählen, damit keine präjudizierende Wirkung entsteht. Speziell bei der Beschreibung von MRI-Bildern muss der Begriff «Riss» durch den Begriff «Signaländerung» ersetzt werden. Denn die Bilder selber geben keine Auskunft über die Entstehung der Signaländerung.

\section{Es hat sich mittlerweile breitflächig herumgesprochen, dass alles unternommen werden muss, den Graubereich der UKS auszunützen.}

tät anzustreben. Der Gedanke kann heute nicht verdrängt werden, dass die beabsichtigte Erleichterung bei der Anerkennung eines Anspruchs auf UVG-Leistungen letztlich zu einem grösseren und mühseligeren Aufwand bei der späteren Begrenzung der Leistungen geführt hat.

In der Gesamtbilanz erscheinen in heutiger Betrachtung die Folgen des «Zwischendings» UKS im medizinischen Berufsalltag problematisch und geben angesichts der geschilderten Störfelder Anlass, über Entscheidungsgrundlagen nachzudenken, welche die pathomorphologischen und rudimentären traumabiomechanischen Aspekte miteinbeziehen.

Anlässlich eines Fortbildungstages des Schweizerischen Versicherungsverbandes in Olten am 17. November 2011 machte der renommierte deutsche Unfallchirurge und Biomechaniker Uwe Moorahrend [3] prüfenswerte Vorschläge für sinnvollere Entscheidungskriterien, ob ein Ereignis Unfallrelevanz erlangt hat oder nicht. Hieraus wären folgende Fragen einer modifizierten Checkliste zu prüfen:

1. War die Einwirkung auf das Gelenk unvorbereitet?

2. Gab es eine exzentrische Gelenkbelastung?

3. War die Reaktionsfähigkeit des Geschädigten intakt?

4. Traten sofort Schmerzen nach dem Ereignis auf?

5. Entstand ein sofortiger Fähigkeitsverlust nach dem Ereignis?

6. Ist das Gelenk im Röntgen unauffällig?

7. Weist die Anamnese auf keine früheren Knieschmerzen hin?

Je mehr Fragen mit Nein beantwortet werden, umso eher ist eine UKS abzulehnen. Dieser Vorschlag soll einstweilen als richtungweisend, aber noch nicht als abschliessend betrachtet werden.
- Bei jeder Erhebung der Anamnese und der klinischen Untersuchung sind ausreichende Angaben über den Hergang, die Pathomorphologie sowie die Leistungsfähigkeiten des Patienten vor und unmittelbar nach dem Ereignis anzugeben.

- Der Einsatz einer einfachen Checkliste analog der oben erwähnten von Moorahrend sollte zumindest für eine Versuchsperiode getestet werden.

- Die bewusste Manipulation der medizinischen Sachlage zwecks ökonomischer Vorteilnahme durch jeden Beteiligten ist unethisch. Es wäre zu wünschen, dass die unterschiedlichen finanziellen Anreize durch die verschiedenen Versicherungssysteme irgendeinmal verschwinden.

\section{Literatur}

1 Bühler A. Meniskusläsionen und soziale Unfallversicherung. Schweiz Ärztezeitung. 2001;82(44): 2339-42.

2 Niederberger O, Stutz K. Wann liegt nach neuester Rechtsprechung des EVG eine unfallähnliche Körperschädigung (UKS) vor - auch an der Rotatorenmanschette? Schweiz Ärztezeitung. 2002;83(20):999_ 1000.

3 Moorahrend U. Vortrag Ärztetagung SVV. 17. November 2011. Olten.

\section{Interaktiver Artikel}

Wollen Sie diesen Artikel kommentieren? Nutzen Sie dafür die Kommentarfunktion in der OnlineVersion oder sehen Sie nach, was Ihre Kolleginnen und Kollegen bereits geschrieben haben: www.saez.ch/aktuelle-ausgabe/interaktive-beitraege/ 